\title{
Patient Centricity: Can PROMs Fill the Gap between the Physician Perspective and the Dynamic Pattern of Patient Perceived Remission in Rheumatoid Arthritis
}

\author{
El Miedany $\mathrm{Y}^{1,2^{*}}$, El Gaafary $\mathrm{M}^{3}$, El Aroussy $\mathrm{N}^{2}$ and Youssef $\mathrm{S}^{2}$ \\ ${ }^{1}$ Rheumatology, Darent Valley Hospital, Darenth Wood Road, Dartford, DA2 8DA, England, United Kingdom \\ ${ }^{2}$ MD, Rheumatology \& Rehab, Ain Shams University, School of Medicine St. Abbassia, 11381, Cairo, Egypt \\ ${ }^{3}$ MD, Community and Public Health, Ain Shams University, School of Medicine St. Abbassia, 11381 Cairo, Egypt
}

Received: April 09, 2018; Accepted: April 30, 2018; Published: May 07, 2018

*Corresponding author: Y. El Miedany, MD FRCP, Darent Valley Hospital, Dartford, Kent, DA2 8DA, UK. E-mail: drelmiedany@rheumatology4u.com

\begin{abstract}
Objective: 1 . Identify whether there is a discordance on comparing the patient versus the rheumatologist perspective on remission in RA; and, 2. Determine, from the patient perspective, the relation of patient reported outcomes to the most prevalent specific disease aspects reported in RA patients who achieved remission.

Methods: Early RA patients diagnosed according to ACR/EULAR criteria were treated according a treat-to-target regime with regular disease activity monitoring. Remission was measured as: 1) patient perceived-; and 2) Physician perceived-remission. The study included 188 RA patients and 87 rheumatologists. All participants were asked to complete a survey composed of all domains identified in relation to the disease remission. $10 \mathrm{~cm}$ visual analogue scale was used to illustrate the importance of each domain.

Results: The top 4 domains reported in the patients' cohort who achieved remission were: pain (76\%), functional ability $(71 \%)$, quality of life $(69 \%)$ and fatigue $(43 \%)$, whereas the top 4 in the rheumatologist cohort were: low disease activity score (88\%), radiologic remission and progression of erosions (76\%), lab measures (ESR, CRP) (57\%) followed by work difficulties (49\%). There was a dynamic pattern of the most important domains reported when patients are stratified according to age, disease duration, comorbidity and motivation.
\end{abstract}

Conclusion: Different factors are important for rheumatologists and RA patients regarding disease remission. Treatment satisfaction is determined not only by disease activity indices but also by other patient-oriented factors. PROMs could optimise targeted therapy as it can play a significant role in identifying disease activity parameters relevant to both the treating rheumatologist as well as the patient.

Keywords: Discordance; PROMs; Rheumatoid arthritis; Patient perceived remission; Physician perceived remission

\section{Introduction}

The significant attainments in inflammatory arthritis management and the recent views whether a stage of cure can be achieved, highlighted the need to identify and characterize remission in rheumatoid arthritis (RA) [1-5]. Over the past few years, remission has been recognized as the major and recommended target of treatment $[6,7]$, and once achieved, it may be possible to optimize the patient's treatment $[8,9]$. The Patient Perspective Workshop at OMERACT [10] addressed the question of assessing the outcomes of intervention in RA from the perspective of those who experience the disease, and the necessary work required to include the patient perspective outcome assessments in the internationally agreed RA "core set" $[11,12]$. The changing concept of the patients who moved from recipients to contributors, and got engaged in their own healthcare decision-making process, warranted further change in the RA management recommendations and guidelines to adopt strategies endorsing patient centered approach in standard clinical practice. The phrase "patient centricity" was recently defined in an article published in the BMJ Innovations [13] as "Putting the patient first in an open and sustained engagement of the patient to respectfully and compassionately achieve the best experience and outcome for that person and their family."

In contrast to many chronic diseases, where a single "gold standard" measure, such as blood pressure in hypertension, haemoglobin $\mathrm{A} 1 \mathrm{C}$ in diabetes, and lipid profile in hyperlipidemia etc., is applicable to diagnosis, management, prognosis, and analyses of outcomes in all individual patients in clinical trials, clinical care, and long-term databases; in inflammatory arthritic conditions (excluding gouty arthritis), there is not a single "gold standard" measure to assess outcomes. This is applicable both in short-term trials such as joint and laboratory measures, as well as in long-term studies such as radiographic progression, disability, and death. The absence of such a gold standard measure highlighted the need for pooled indices as a valid tool $[14,15]$. However, the most commonly used tools to monitor disease activity and identify remission do not endorse the concept of patient reported remission satisfactorily. For example, disease activity score (DAS-28) [16] includes only 
patient global assessment in addition to tender and swollen joints as well as inflammatory marker measure. Similarly, the ACR/ EULAR remission definition [17] included only patient global assessment, based on the strong correlation between patient global assessment and pain. Furthermore, apart from physical functioning, no other patient reported outcome was available in the validation phase of this definition.

There is a current need to evaluate the patients' perspective of disease remission and how and which aspects of disease activity measures are most profound to them. There is also a need for further information regarding the performance of variable combinations of patient reported outcomes in relation to remission in RA. Since remission is relatively a new treatment target, and knowledge on the patients' perspective on remission is limited, there is requirement for identifying specific and sensitive outcome measures for remission in RA patients. This study was carried out aiming at: 1 . Identify whether there is a discordance on comparing the patient versus the rheumatologist perspective on remission in RA; and, 2. Determine, from the patient perspective, the relation of patient reported outcomes to the most prevalent specific disease aspects reported in RA patients who achieved remission.

\section{Methods}

\section{Study Design}

This was a retrospective cohort study, which included 188 early RA patients diagnosed according to the ACR/EULAR criteria [18] who achieved sustained disease remission, and 188 who have not achieved the disease remission status. The patients were followed longitudinally from baseline for an average of 36 months duration.

\section{Ethical Matters}

Local ethical and methodological protocols for approval of the study were followed. All patients who participated in the study signed an informed consent according to the Declaration of Helsinki (at the General Assembly in October 2008).

\section{Patients}

Patients enrolled in this database were diagnosed, treated and monitored regularly in the outpatient rheumatology clinics according to a treat-to-target regimen. Information on demographic features and all core set measures were regularly recorded. These data included age, sex, disease duration (defined as time since diagnosis), and disease activity score (DAS-28) [16], presence of rheumatoid factor and/or anti- citrullinated protein antibodies (ACPAs), as well as inflammatory markers: the C-reactive protein (CRP) level and erythrocyte sedimentation rate (ESR).

\section{Medical Management}

On confirming the diagnosis, DMARD(s) therapy was commenced following EULAR guidelines [19], adopting shared decision approach [20]. According to guidelines, a combination of disease-modifying anti-rheumatic drugs (DMARDs) (including methotrexate and at least one other DMARD, plus short-term glucocorticoids) is offered as first-line treatment. In people for whom combined DMARD therapy is not appropriate, DMARD monotherapy was started, placing greater emphasis on fast escalation to a clinically effective dose rather than on the choice of DMARD. Biologic therapy was commenced for those patients whose disease remained active (RA: DAS-28 > 5.1) after 6-month of DMARDs therapy. Monitoring the disease (using a composite score such as DAS-28) was carried out initially monthly then 3 -monthly until treatment has controlled the disease activity.

At baseline and after 12, 24, 36 and 52 weeks of treatment PROMs as well as Disease Activity score (DAS-28) were assessed and recorded.

Patients who achieved remission and remained in remission for 6-months (sustained remission), were included in this work.

\section{Definition of Remission}

\section{Remission was measured in 5-ways}

1. Patient perceived remission using the question "Would you say that, at this moment, your disease activity is as good as gone? (yes/no)"; and

2. Physician perceived remission was defined as a physician global assessment $\leq 1$ on a visual analogue scale (0-10 VAS), phrased: "How active do you think the rheumatoid arthritis of your patient is today?"

3. ACR/EULAR Boolean-based definition of remission [17] with swollen joint count, tender joint count, patient global assessment as well as CRP all $\leq 1$.

4. DAS-28 score $<2.6$ and CDAI $<2.8$ (CDAI=swollen joint count + tender joint count + physician global assessment + patient global assessment)

All patients who achieved remission and included in this study, met these 5 criteria.

\section{The Survey}

The survey items were based on domains remission identified in a previous study [21]. 20 items were included representing the most important items recognized by the patients which reflect remission according to RA patient's perspective. The list included joint pain, joint swelling, sleep quality, ability to do activities of daily living, ability to work, independence, functional ability, quality of life, absence of morning stiffness, absence of fatigue, socialize, feel anxious, feel normal, feel down, family role, normal laboratory tests, no comorbidity risk, radiologic remission, Disease Activity score, doctors view. To determine the level of importance of the different domains, every patient was asked to rate each domain on a Visual analogue scale (scored 0-10) to illustrate the importance of each factor in an individual opinion. The power of the domain was assessed by taking the mean of the rates reported by the patients.

The survey was completed by 188 RA patients who were in remission, as well as $188 \mathrm{RA}$ whose disease remained active (DAS-28 >3.2). 61-rheumatologists were also asked to complete the RA remission questionnaire, from their perspectives. 


\section{Validity of the Proms to Assess the Patient Perceived Remission}

To assess for the validity of PROMs and its predictive ability of remission, prior to each clinic visit every patient included in this study was asked to complete a copy of the patient reported outcome measures [21]. Outcomes reported by the patients who achieved remission included in this work were compared to outcomes of a control group of 188 RA patients who have not achieved remission.

\section{Statistical Analysis}

One-way analysis of variance was used for the comparison of independent variables. Spearman correlation coefficient was used to assess the correlation between variables. Cluster analysis was performed to identify items for which respondents gave similar answers. Multivariable logistic regression model was used to assess the determinants of patient global score $<1$.

\section{Results}

\section{Demographics and Clinical Characteristics}

The study included 188 rheumatoid arthritis patients (76 males, 112 females; mean age $52.4 \pm 11.7$ years; range 28 to 78 years) and 61 rheumatologists (29 males, 32 females; mean age $48.7 \pm 11.7$ years; range 33 to 67 years, mean duration of work as rheumatologists $9.4+4.3$ years).Mean disease duration was 19.3 \pm 8.9 months; whereas mean disease activity score (DAS-28) was $1.7 \pm 0.6$. The mean age of the control group was $53.1 \pm 10.4$ years, range 26- 79 years; with disease duration of $19.5 \pm 9.1$ months and mean DAS-28 score of $4.8 \pm 4.2$. Table 1 shows a summary of the demographic and clinical characteristics of the rheumatoid arthritis patients included in this study. [Table 1]

Table 1: Demographic and clinical characteristics of the rheumatoid arthritis patients included in this study.

\begin{tabular}{|c|c|c|}
\hline Parameter & Study Group (188 patients) & Control group (118 patients) \\
\hline Male: Female & $76: 112$ & $75: 113$ \\
\hline Age (Mean \pm S.D) years & $52.4 \pm 11.7$ & $53.1 \pm 10.4$ \\
\hline Disease duration (Mean \pm S.D) months & $19.3 \pm 8.9$ & $19.5 \pm 9.1$ \\
\hline DAS-28 score(Mean \pm S.D) & $1.7 \pm 0.6$ & $4.8 \pm 4.2$ \\
\hline Duration of morning stiffness (Mean \pm S.D) minutes & $5.2 \pm 3.7$ & $42 \pm 76$ \\
\hline Functional Disability (score 0-3) & $0.1 \pm 0.3$ & $1.1 \pm 0.8$ \\
\hline Quality of life (score $0-3)($ Mean \pm S.D) & $0.1 \pm 0.2$ & $1.2 \pm 0.9$ \\
\hline Patient Global score (VAS 0-10) (Mean \pm S.D) & $0.6 \pm 0.3$ & $5.3 \pm 1.4$ \\
\hline Physician Global score (VAS 0-10)(Mean \pm S.D) & $0.5 \pm 0.3$ & $5.9 \pm 1.6$ \\
\hline ESR(Mean \pm S.D) $\mathrm{mm} / \mathrm{hr}$ & $8.2 \pm 2.6$ & $31.6 \pm 14.3$ \\
\hline Rheumatoid factor (\%) & $151 / 188(80.3 \%)$ & $153 / 188(81.4 \%)$ \\
\hline Anti-CCP (\%) & $124 / 188(66 \%)$ & $123 / 188(65.4 \%)$ \\
\hline Number of patients on DMARDs (\%) & $139 / 188(74 \%)$ & $138 / 188(73.4 \%)$ \\
\hline Number of patients on biologics (\%) & $49 / 188(26 \%)$ & $50 / 188(26.6 \%)$ \\
\hline
\end{tabular}

\section{Patients Vs Physician Perceived Remission}

There was no significant difference on comparing the rheumatologists highly rated domains to RA patients who have not achieved remission. Joint pain, swelling, functional ability and quality of life were rated as the highest by both the patients, whereas joint pain, swelling, disease activity score and functional ability were the most highly rated domains reported by the rheumatologists. There was significant discrepancy between the rheumatologists and RA patients who achieved remission scores. Figure 1, summarizes the most important domains rated the different patient groups as well as the rheumatologists. [Figure $1 \mathrm{~A}, 1 \mathrm{~B}]$

\section{The Dynamic Nature of the Patient Perceived Remission}

Stratifying the RA patients who achieved remission according to different dominant factors revealed:

Age: Cut-off point 65-years old.
Patients $<65$ scored functional ability significantly higher than those $>65$ years of age ( 9.3 Vs 8.1 on VAS, $p=0.03$ ). Similarly work ability was scored higher in the patients below 65 than those above 65 (8.8 Vs 5.1 on VAS, p< 0.05)

Patient's cohort $>65$ years of age, rated quality of life at a higher level (9.4 Vs 8.4) than those $<65$ years old. Likewise, independence was rated higher in the cohort of patients $>65$ years old (8.4 Vs 6.7 on VAS, $p<0.05$ )

There was no significant difference in pain and fatigue scores between patients' cohorts classified by age.

Disease duration: Cut off point 2-years

Mean level of importance scores of functional ability ( 9.3 Vs 8.6) and independence (9.1 Vs 8.1) were significantly higher in patients with longer-standing disease ( $>2$-years) as compared to patients with shorter disease duration $(<2$-years) $(\mathrm{p}<0.05)$, 


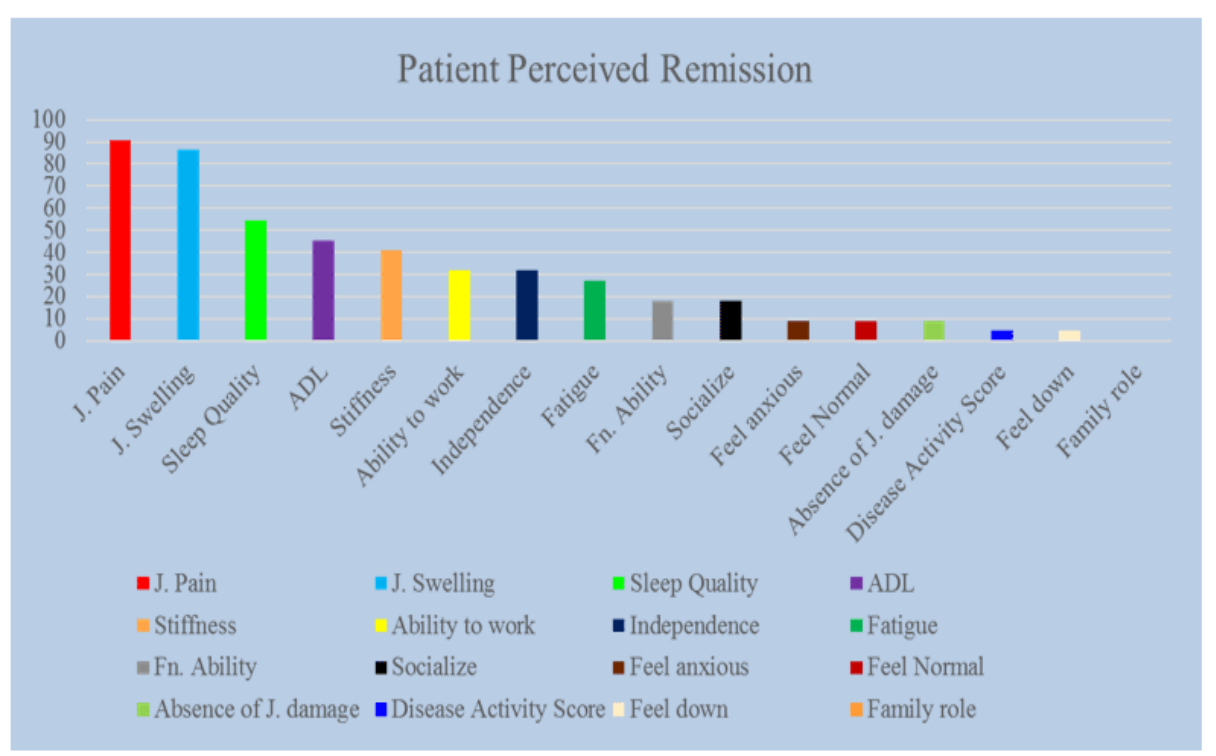

Figure 1A: Reported measures in the patient perceived remission cohort.

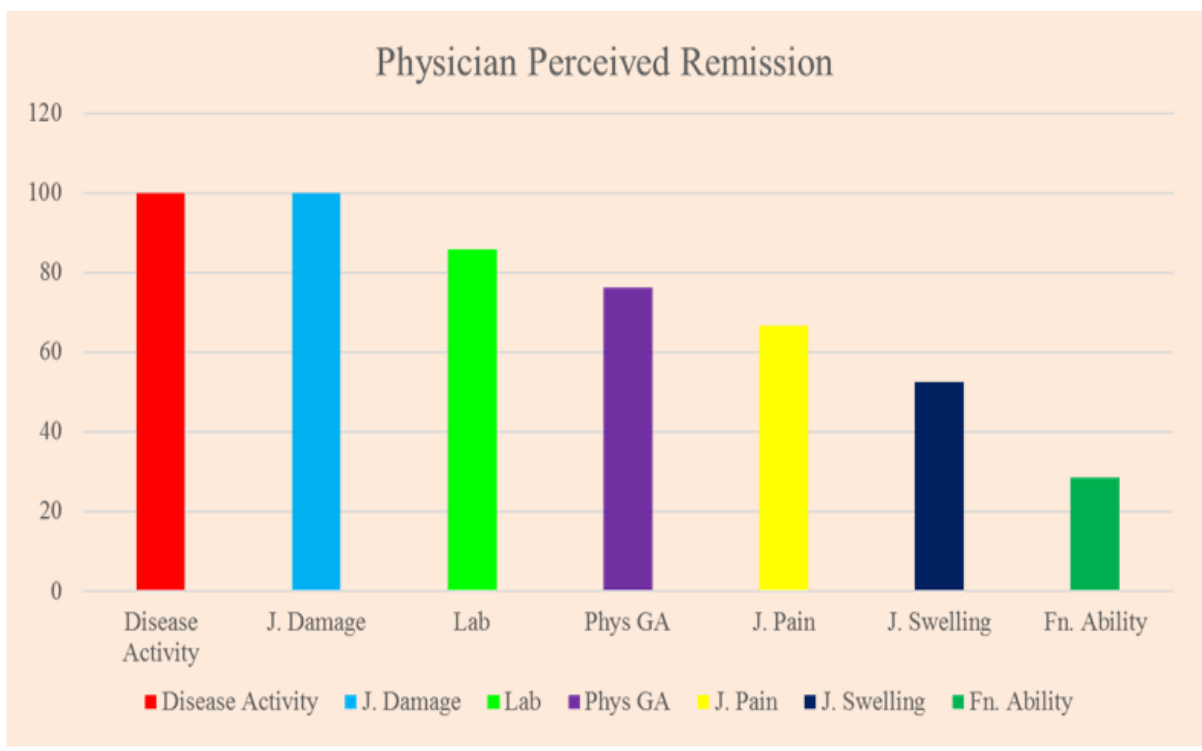

Figure 1B: measures in the physician perceived remission cohort.

whereas quality of life was rated higher amongst patients with shorter disease duration (8.9 Vs 7.8).

Patients with low Motivation rated high: Depression, anxiety, support, independence, ability to work, functional ability

\section{Sustained Remission Vs Deep Remission}

Sustained remission was defined as meeting the remission criteria for 6-months consecutively, whereas deep remission was defined as DAS-28 $<2$.

Ability to work, feel normal and ability to socialize were all rated higher in the cohort of patients whose arthritis is in deep remission in contrast to those who were in sustained remission (mean scores were 9.3 Vs 8.8, 9.6 Vs 9.1 and 8.9 Vs 8.1 respectively).

Table 2 shows the discordance of the top rated 3 domains and its scores amongst the RA patients included in this study, who achieved remission, when stratified according to the commonest variables. [Table 2]

\section{Validity of PROMs}

PROMs enabled the assessment and scoring of the different domains recognized by the patients as influential parameters to identify remission, hence enable both the patient and treating 
Table 2: Discordance of the top rated 3 domains and its scores (Mean \pm S.D) amongst the RA patients included in this study, who achieved remission, when stratified according to the commonest variables.

\begin{tabular}{|c|c|c|c|c|c|c|c|c|c|c|}
\hline \multirow[b]{2}{*}{ Stratification } & \multicolumn{2}{|c|}{ Age (years) } & \multicolumn{2}{|c|}{ Disease Duration (years) } & \multicolumn{2}{|c|}{ Disease activity (DAS-28_ } & \multicolumn{2}{|c|}{ Comorbidity } & \multicolumn{2}{|c|}{ Motivation (score out of 10 ) } \\
\hline & $<65$ & $>65$ & $>2-y r$ & $<2-\mathrm{yr}$ & $<2$ & $2-2.6$ & $<2$ & $>3$ & $<1$ & $>6$ \\
\hline & $9.4 \pm 0.2$ & $9.4 \pm 0.3$ & $9.3 \pm 0.3$ & $8.9 \pm 0.4$ & $9.3 \pm 0.3$ & $9.2 \pm 0.3$ & $9.1 \pm 0.4$ & $9.4 \pm 0.3$ & $9.5 \pm 0.3$ & $9.6 \pm 0.3$ \\
\hline & $8.8 \pm 0.4$ & $8.4 \pm 0.3$ & $9.1 \pm 0.3$ & $8.7 \pm 0.5$ & $9.1 \pm 0.4$ & $8.9 \pm 0.4$ & $9.1 \pm 0.3$ & $9.2 \pm 0.2$ & $9.1 \pm 0.3$ & $9.5 \pm 0.4$ \\
\hline & Socialize & Family role & Stiffness & $\begin{array}{c}\text { Feel } \\
\text { normal }\end{array}$ & Socialize & ADL & Family Role & Anxious & Work ability & ADL \\
\hline & $8.7 \pm 0.4$ & $8.6 \pm 0.5$ & $8.7 \pm 0.6$ & $8.7 \pm 0.5$ & $8.9 \pm 0.5$ & $8.8 \pm 0.6$ & $8.9 \pm 0.5$ & $8.9 \pm 0.5$ & $8.9 \pm 0.3$ & $9.3 \pm 0.3$ \\
\hline
\end{tabular}

Fn. Ability: Functional Ability, QoL: Quality of Life

Table 3: Multivariable logistic regression model of determinants of patient global score <1 in 188 patients

\begin{tabular}{|c|c|c|}
\hline Multivariate Logistic regression & OR to achieve PtGA<1 & 95\% CI \\
\hline HAQ improvement in first 3month* & 3.5 \\
\hline Patient motivation improvement in the first 6-months* & 2.9 \\
\hline QoL improvement in first 3month* & 2.8 \\
\hline MS improvement in the first 6month* & $1.2-6.5$ \\
\hline Fatigue score improvement in the first 6 months* & 1.9 & 1.8 \\
\hline $\begin{array}{l}\text { *p<0.05 } \\
\text { HAQ: Health Assessment Questionnaire, QoL: Quality of Life, MS: Morning Stiffness }\end{array}$ & $2.1+2-7.4$ \\
\hline
\end{tabular}

doctor to assess for the disease activity status. Patients in selfperceived remission had a significantly improved functional disability, QoL, pain, fatigue, morning stiffness as well as motivation score at week $12(\mathrm{p}<0.001)$ and week $24(\mathrm{p}=0.007)$ compared to patients not in self-perceived remission

The multivariable linear regression showed that significant improvement ( $>50 \%$ ) of functional disability, motivation, quality of life and fatigue were independent predictors of disease activity remission as well as patient reported global score $<1$. [Table 3]

\section{Discussion}

Patient centricity is defined as the process of designing a service with the patient centred in its core. Though the concept sounds easy to grasp, the scope and pace are slow and narrow, especially for patients with chronic conditions. Patients with chronic conditions interact with the healthcare system for an average of $2 \%$ of the entire year, and they are left "self-centric" to manage their own condition for the remaining 98\% [22]. For patient centricity to be fully embraced in day to day practice, there are several barriers need to be overcome. The challenges come from the belief amongst some of the health care professionals that talking to patients is not as helpful as examining and viewing the blood results or x-rays, whereas others consider patients' input "subjective and non-reproducible," which, makes it something to be overlooked or ignored [23]. This has been reflected onto the physicians' perspective of disease flare and remission and might explain the discrepancy noted in this study as well as other studies. Another barrier is that patients' perceptive have not been fully considered in the disease activity tools commonly used to monitor the patients' disease activity and guide treatment pathways [24].

Results of this study revealed discrepancies in the patients' perspective, in comparison to the Rheumatologists' perception, of evaluating their disease activity status and identifying the main themes of rheumatoid arthritis remission. There were also discrepancies amongst RA patients themselves, comparing those who achieved remission to those who have not. These findings agree with earlier published data[25]reporting that whilst 'Staying independent', 'reducing pain', and 'keeping mobile' were the most frequently selected by RA patients who achieved remission as the top 3 (by 39\%, 36\% and 34\% respectively)and are already in the American College of Rheumatology (ACR) core set; others such as 'returning to/ maintaining a normal lifestyle', 'feeling well in myself' and 'enjoying my life' which were also commonly selected (by 26\%,20\%and 18\% respectively), are not in the ACR core set or disease activity score (DAS). In another study [26], the "RA Impact of Disease (RAID) index", a patient-derived composite response index developed by a EULAR initiative, pain(21\%), functional disability (16\%), fatigue (15\%), emotional well-being $(12 \%)$, sleep (12\%),coping (12\%), and physical well-being $(12 \%)$ [12] Were identified by the patients as the most important domains expressing their concept of remission. In a third study 
[27], outcomes revealed that the discordance between patient's and evaluator's assessment of disease activity may reduce the likelihood of remission across the RA and PsA diagnoses as well as across different treatment regimens. These data suggest that patient priorities only partly overlap with those included in the core sets developed by health professionals. It also highlights the importance of including domains identified by the patients, representing their disease activity status, as part of the standard clinical practice. In addition to its role in the patient management, this will also improve communication and establish a real doctorpatient partnership towards successful management.

The dynamic nature of the domains rated by RA patient who achieved remission, identified in this study, endorse the importance of consistent recording and monitoring of these measures on regular basis during the patient's management course. Results of this study agree with the earlier study done by van Tuyl et al [28] where some differences were reported between different patient groups when stratified for age, gender, disease duration, the presence of comorbidities and joint damage. Whilst performing one's family role was more relevant and reported imported amongst younger patients, older patients rated functional ability and feeling independent of higher importance. In concordance, outcomes of this work revealed that functional ability was rated higher in patients with longer-standing disease as compared to patients with shorter disease duration, whereas patients with low motivation rated depression, anxiety, support, independence, ability to work, functional ability as the highest. These findings come in concordance with earlier published data which reported that whilst most available analysis of patientphysician discordance identified pain, physical function and health-related quality of life as potential predictive factors of discordance, in RA; psychological aspects and to a lesser degree, fatigue, remain rarely reported in clinical trials and cohorts of RA patients [29]. This was supported by the outcomes of another study [30] which attributed that discordance between patients and their treating physicians, to psychological factors rather than physical domains of health amongst RA patients. The study reported that depression was found to be associated with an underestimation by the physician of the patient's symptoms (i.e., pain, anxiety, physical function). Outcomes of this work revealed that the patient's motivation seems to be important, however, this has been overlooked in earlier studies. These findings ratify the importance of considering these measures whilst assessing and managing the patients in the day to day clinical practice. Disagreements in the assessment of the disease activity status are a real problem, with an impact on treatment decisions and treatment outcomes. Regular scoring would be of vital help to identify any significant changes in these domains.

Identifying the most important domains representing the patients' perspective of remission won't be meaningful without a tool to assess and monitor them. Such tools recording the patients' input, should be reproducible, reliable and sensitive to change. Results of this work revealed that PROMs could fill this gap in the patients' management. In comparison to the patients not in self-perceived remission, those in self-perceived remission had significantly improved changes in functional disability, quality of life, fatigue as well as patient motivation scores. PROMs offer the potential to expand the disease activity/ remission core set in standard clinical practice, as well as longitudinal observational studies, improving the incorporation of the patient perspective, and including disease impacts such as fatigue and patient motivation as a core criterion. The dynamic nature of PROMs has been depicted in earlier study [31]. Results of that work revealed that the dynamic role helps in driving improvement not only in the quality of inflammatory arthritis care but also in the patients' reported experience. Therefore, in addition to its value in tailoring treatment targets adapted to the patient's needs, PROMs also have the potential of modifying the disease impact through improving the patients' adherence to therapy and allowing the patients to monitor the changes in their condition.

\section{Conclusion}

In conclusion, this study brings important information on the gap between patient and physician assessment in RA. The discordance was more frequent in patients in remission which reflect different patients' expectations regarding their disease status compared to their treating physicians.

PROMs enabled the patient and the treating physician identifies the aspects of relevance necessary for optimal clinical management. Treatment strategies aiming at remission should consider the patient perceived remission in their pathway. Discordance may hamper shared decision-making.

\section{Competing Interest}

The authors have no relevant financial disclosures. No financial conflict of interest in relation to the study to be disclosed.

\section{Contributorship}

All authors had a substantial contribution to the conception and design of the study, drafting/ revising of the article and final approval of the version to be published.

\section{Acknowledgements}

We express thanks to all participants, our colleagues, research assistants and nurses for their cooperation and help to bring this research to its final conclusions.

\section{References}

1. McPherson KM, Brander P, Taylor WJ, McNaughton HK. Living with arthritis -- what is important? Disabil Rehabil. 2001; 23(16): 706721.

2. Carr A, Hewlett S, Hughes R, Mitchell H, Ryan S, Carr M, et al. Rheumatology outcomes: The patient's perspective. J Rheumatol. 2003; 30(4): 880-883.

3. Ahlmen M, Nordenskiold U, Archenholtz B, Thyberg I, Ronnqvist R, Linden L, et al. Rheumatology outcomes: The patient's perspective. A multicentre focus group interview study of Swedish rheumatoid arthritis patients. Rheumatology. 2005; 44(1): 105-110. DOI:10.1093/rheumatology/keh412 
4. Marshall NJ, Wilson G, Lapworth K, Kay LJ. Patients' perceptions of treatment with anti-TNF therapy for rheumatoid arthritis: A qualitative study. Rheumatology. 2004; 43(8):1034-1038. DOI: 10.1093/rheumatology/keh237

5. Edwards J. An exploration of patients experiences of anti-TNF therapy. Musculoskeletal Care. 2004; 2(1): 40-50. DOI: 10.1002/msc.55

6. Kalyoncu U, Dougados M, Daures J, Gossec L. Reporting of patientreported outcomes in recent tirals in rheumatoid arthritis: A systematic literature review. Ann Rheum Dis. 2009; 68(2):183-190. DOI:10.1136/ard.2007.084848

7. Felson DT, Anderson JJ, Boers M, Bombardier C, Chernoff M, Fried B, et al. The Amercian College of rheumatology preliminary cores set of disease activity measures for rheumatoid arthritis clinical trials. Arthritis Rheum. 1993; 36(6):729-740.

8. van der Heijde D, Hof M, Piet L, Putte L. Development of a disease activity score based on judgement in clinical practice by rheumatologists. J Rheumatol. 1993; 20(3): 579-581.

9. El Miedany Y, El Gaafary M, Youssef S, Ahmed I, Bahlas S, Hegazi M, et al. Optimizing therapy in inflammatory arthritis: prediction of relapse after tapering or stopping treatment for rheumatoid arthritis patients achieving clinical and radiological remission. Clin Rheumatol. 2016; 35(12):2915-2923. DOI:10.1007/s10067-016-3413-8

10. Kirwan JR, Ahlmén M, de Wit M, Heiberg T, Hehir M, Hewlett $S$, et al. Progress since OMERACT 6 on including the patient perspective in rheumatoid arthritis outcome assessment. J Rheumatol. 2005; 32(11):2246-2249.

11. Kirwan J, Heiberg T, Hewlett $S$, Hughes R, Kvien T, Ahlmèn $M$, et al. Outcomes from the Patient Perspective workshop at OMERACT 6. J Rheumatol. 2003; 30:868-872.

12. Boers M, Tugwell P, Felson DT, van Riel PL, Kirwan JR, Edmonds JP, et al. World Health Organization and International League of Associations for Rheumatology core endpoints for symptom modifying anti rheumatic drugs in rheumatoid arthritis clinical trials. J Rheumatol. 1994; 21 Suppl 41:86-89.

13. Yeoman G, Furlong P, Seres M, Helena Binder, Helena Chung, Vincenzo Garzya, et al. Defining patient centricity with patients for patients and caregivers: a collaborative endeavor. BMJ Innovations Published Online First: March. 2017. DOI: 10.1136/bmjinnov-2016-000157

14. Tugwell P, Bombardier C. A methodologic framework for developing and selecting endpoints in clinical trials. J Rheumatol 1982; 9:758-62.

15. Bombardier C, Tugwell P. A methodological framework to develop and select indices for clinical trials: statistical and judgmental approaches. J Rheumatol 1982; 9:753-7.

16. Van Der Heijde DMFM, Vant Hof M, Van Riel PLCM, Van De Putte LBA. Development of a disease activity score based on judgement in clinical practice by rheumatologist. J Rheumatol. 1993; 20(3):579-581.

17. Shahouri SH, Michaud K, Mikuls TR, Caplan L, Shaver TS, Anderson JD, et al. Remission of rheumatoid arthritis in clinical practice: Application of the American College of Rheumatology/European League Against Rheumatism 2011 remission criteria. Arthritis Rheum. 2011; 63(11):3204-3215. DOI:10.1002/art.30524

18. Aletaha D, Neogi T, Silman A, Funovits J, Felson D, Bingham CO et al. 2010 Rheumatoid Arthritis Classification Criteria. An American College of Rheumatology/European League Against Rheumatism Collaborative Initiative. Arthritis Rheum. 2010; 62(9): 2569-2581.

\section{DOI:10.1002/art.27584}

19. Smolen JS, Aletaha D, Bijlsma JW, Breedveld FC, Boumpas D, Burmester $\mathrm{G}$ et al. Treating rheumatoid arthritis to target: recommendations of an international task force. Ann Rheum Dis. 2010; 69(4): 631-637. DOI:10.1136/ard.2009.123919

20. El Miedany Y, El Gaafary M, Sayed S, Palmer D, Ahmed I. Implementing Shared Decision Making In Clinical Practice: Outcomes of a New Shared Decision Making Aid for Chronic Inflammatory Arthritis Patients. J Pat Care. 2016; 2(2): 117-123.

21. El Miedany Y, El Gaafary M, Youssef S, Palmer D.Incorporating Patient Reported Outcome Measures in Clinical Practice: Development and Validation of a questionnaire for inflammatory arthritis. Clin Exp Rheumatol. 2010; 28: 734-744.

22. Pincus T, Yazici Y, Sokka T. Quantitative measures of rheumatic diseases for clinical research versus standard clinical care: differences, advantages and limitations. Best Pract Res ClinRheumatol. 2007; 21(4): 601-628. DOI:10.1016/j.berh.2007.02.007

23. Palmer D, El Gaafary M, El Miedany Y. Improving patient care: measurement of outcome in rheumatoid arthritis. Br J Nurs. 2007; 16(16) :1010-1015. DOI:10.12968/bjon.2007.16.16.27081

24. Aletaha D, Machold KP, Nell VPK, Smolen JS. The perception of rheumatoid arthritis core set measures by rheumatologists. Results of a survey. Rheumatology (Oxford). 2006; 45: 1133-1139.

25. Hewlett S, Carr M, Ryan S, Kirwan J, Richards P, Carr A, et al. Outcomes generated by patients with rheumatoid arthritis: How important are they? Musculoskeletal Care. 2005; 3(3):131-142. DOI:10.1002/msc.3

26. Gossec L, Dougados M, Rincheval N, Balanescu A, Boumpas DT, Canadelo $S$, et al. The elaboration of the preliminary rheumatoid arthritis impact of disease (RAID) score: A EULAR initiative. Ann Rheum Dis. 2009; 68(11): 1680-1685. DOI:10.1136/ ard.2008.100271

27. Michelsen B, Kristianslund EK, Hammer HB, Fagerli KM, Lie E, Wierød A et al. Discordance between tender and swollen joint count as well as patient's and evaluator's global assessment may reduce likelihood of remission in patients with rheumatoid arthritis and psoriatic arthritis: data from the prospective multicentre NORDMARD study Ann Rheum Dis. 2017;76(4):708-711. DOI:10.1136/ annrheumdis-2016-210283

28. Van Tuyl LHD, Sadlonova M, Hewlett S, Davis B, Flurey C, Goel N et al The patient perspective on absence of disease activity in rheumatoid arthritis: a survey to identify key domains of patient-perceived remission Ann Rheum Dis. 2017;76(5):855-861. DOI:10.1136/ annrheumdis-2016-209835

29. Kalyoncu U, Ogdie A, Campbell W, Bingham CO, de Wit M, Gladman DD, et al. Systematic literature review of domains assessed in psoriatic arthritis to inform the update of the psoriatic arthritis core domain set. RMD Open. 2016; 2(1):e000217. DOI:10.1136/ rmdopen-2015-000217

30. Zastrow A, Faude V, Seyboth F, Niehoff D, Herzog W, Löwe B. Risk factors of symptom underestimation by physicians. J Psychosom Res. 2008; 64(5):543-551. DOI:10.1016/j.jpsychores.2007.11.010

31. El Miedany Y, El Gaafary M, Palmer D. Assessment of the utility of visual feedback in the treatment of early rheumatoid arthritis patients: a pilot study. Rheumatol Int. 2012; 32(10):3061-3068. DOI:10.1007/s00296-011-2098-1 